\title{
Intrinsic Stepwise Translocation of Stretched ssDNA in Graphene Nanopores
}

Hu Qiu, ${ }^{\dagger}$ Aditya Sarathy, ${ }^{\dagger, \ddagger}$ Jean-Pierre Leburton, ${ }^{*, \dagger,+,}$ and Klaus Schulten ${ }^{*, \dagger}, \boldsymbol{\Phi}$

$\dagger$ †eckman Institute for Advanced Science and Technology

$\ddagger$ Department of Electrical and Computer Engineering

\Department of Physics, University of Illinois, Urbana, Illinois 61801, United States

E-mail: jleburto@illinois.edu; kschulte@ks.uiuc.edu 


\section{Voltage Bias to Translocate and Stretch ssDNA}

DNA translocation can also be induced by applying a voltage bias across the graphene membrane (see Fig. S4). In a previous study ${ }^{1}$ we had shown for the case of a SiN pore that, under the voltage bias, a strong electrostatic potential gradient arises around the narrowest constriction of the pore (see Fig. 4d in Ref. 1), stretching the DNA inside the pore. The stretching can be further enhanced by using a membrane of a p-n conductor. ${ }^{2}$ Accordingly, we propose to employ a multi-layer nanopore device that consists of a monolayer graphene sheet sandwiched between two layers of thicker solid-state material such as $\mathrm{SiO} 2$ and $\mathrm{SiN}$, as depicted in Fig. S5. With this design, DNA can be stretched and translocated simultaneously by the voltage bias. We note that also in the case of a biological nanpore, namely $\alpha$-hemolysin, ssDNA adopts a stretched conformation when translocated by a voltage bias, as illustrated very clearly in a movie showing an MD trajectory of a DNA being translocated through an $\alpha$ hemolysin pore (see http://www.ks.uiuc.edu/Research/hemolysin/translocationDNA.mpg). 


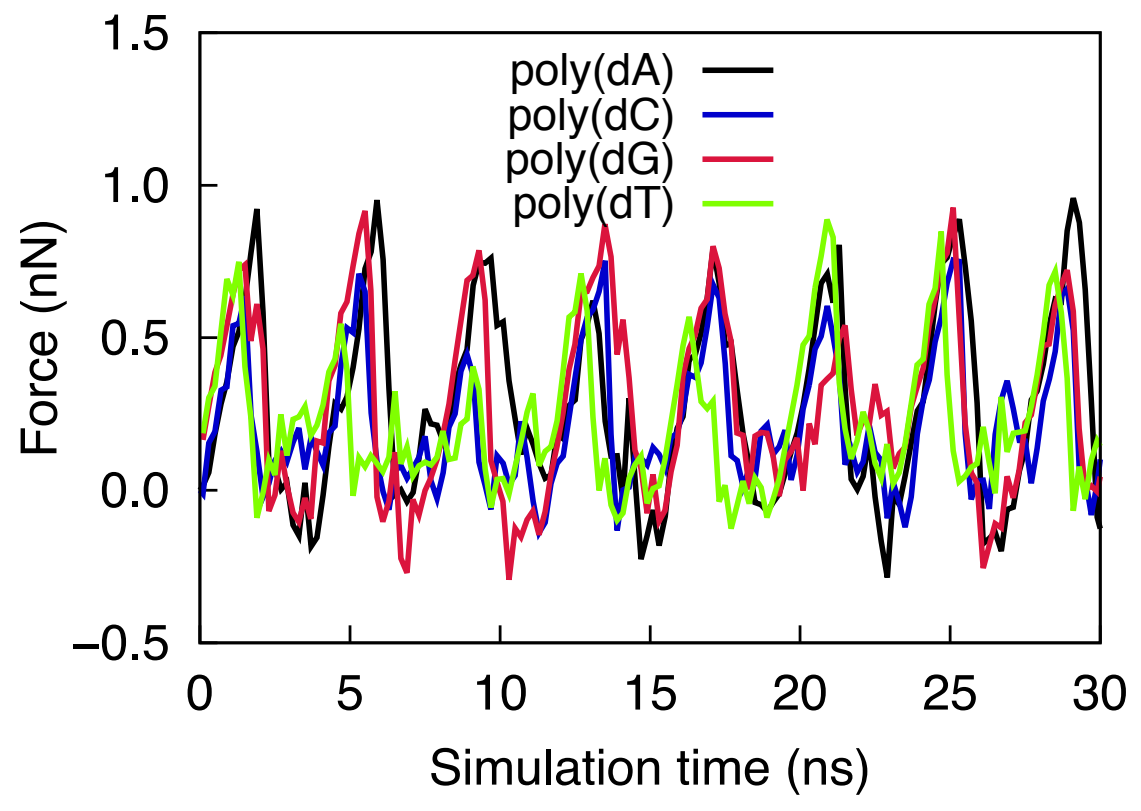

Figure S1: ssDNA pulling forces for poly $(\mathrm{dA})$, poly $(\mathrm{dC}), \operatorname{poly}(\mathrm{dG})$ and poly $(\mathrm{dT})$. This figure presents the pulling force for four types of ssDNA translocated through a $1.6 \mathrm{~nm}$ pore at a speed of $2 \AA /$ s. It supplements the results shown in Fig. $3 \mathrm{~b}$. 

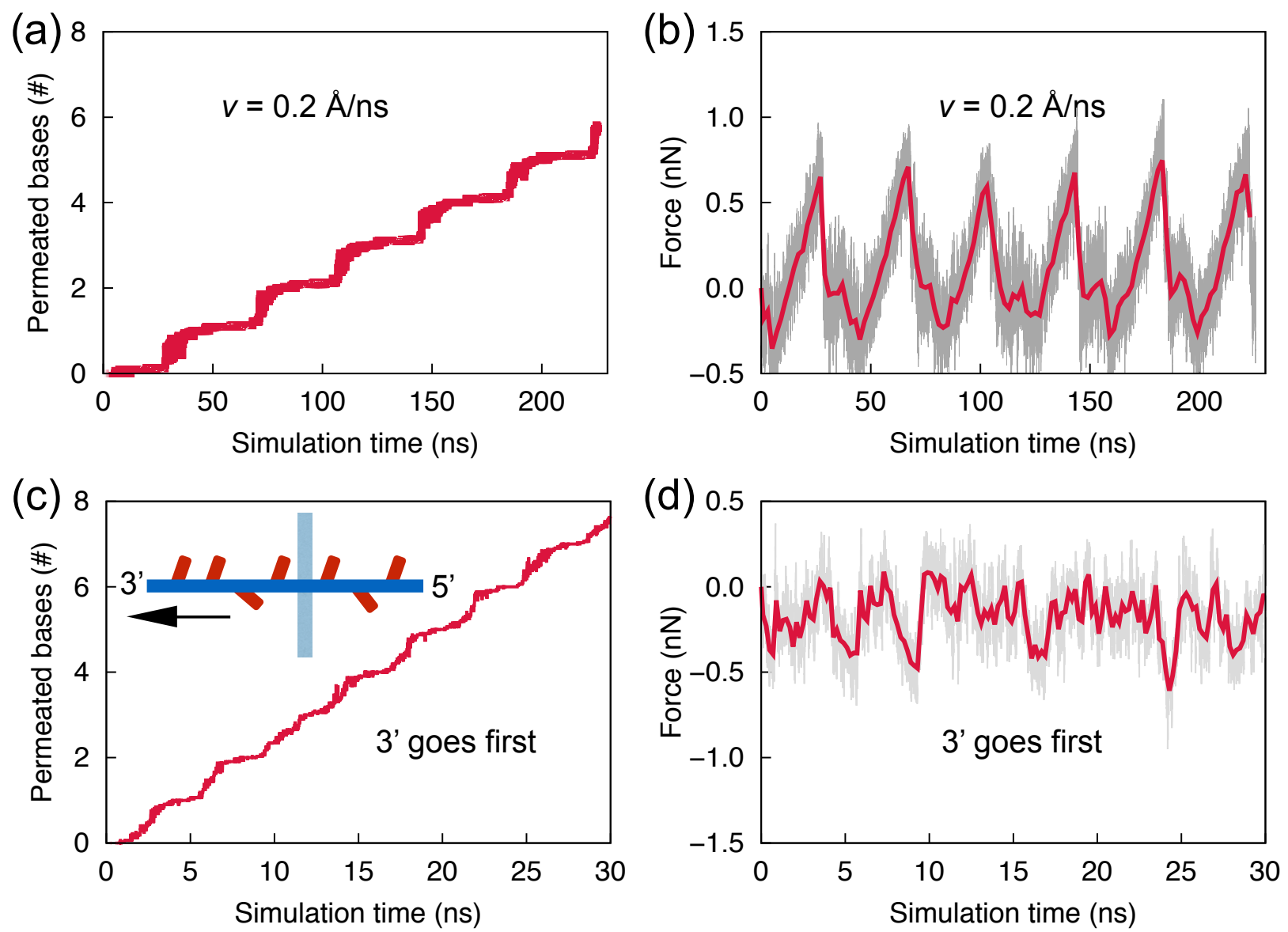

Figure S2: Permeability (left panels) and force signal (right panels) as ssDNA is pulled $5^{\prime}$ end first through a graphene nanopore at a velocity of $v=0.2 \AA / \mathrm{s}[(\mathrm{a}),(\mathrm{b})]$ or in the opposite orientation, namely $3^{\prime}$ end first $[(\mathrm{c}),(\mathrm{d})]$. The data reported in the main text were determined when pulling ssDNA $5^{\prime}$ end first at a velocity of $v=2 \AA / \mathrm{s}$. 
(a)

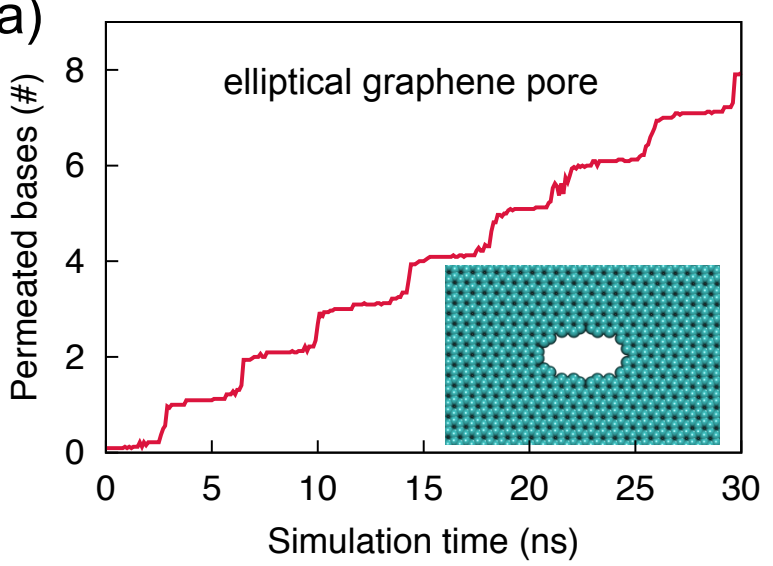

(c)

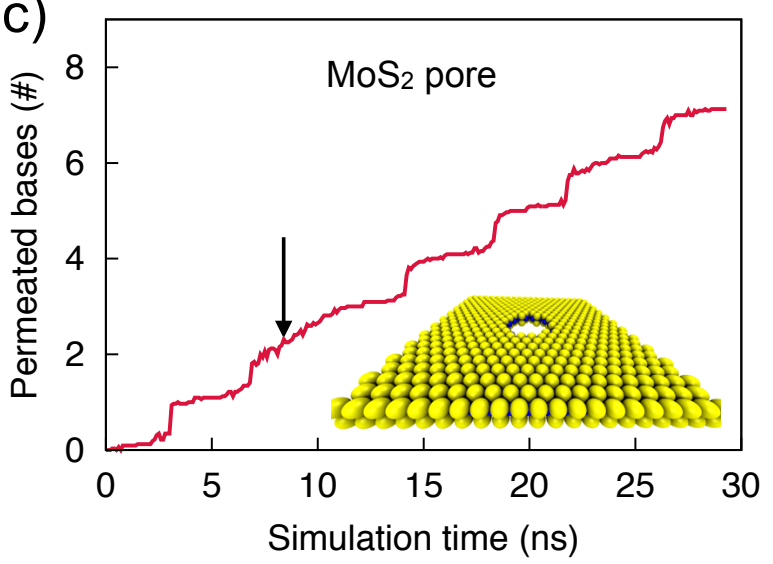

(e)

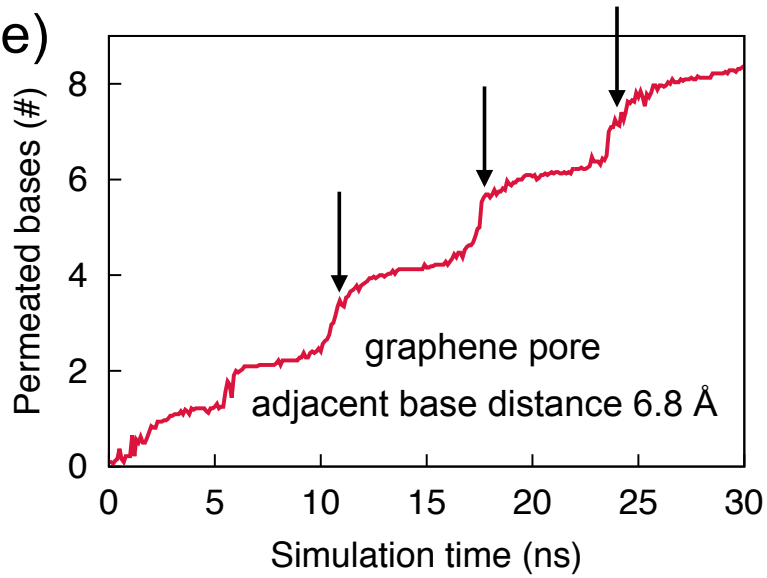

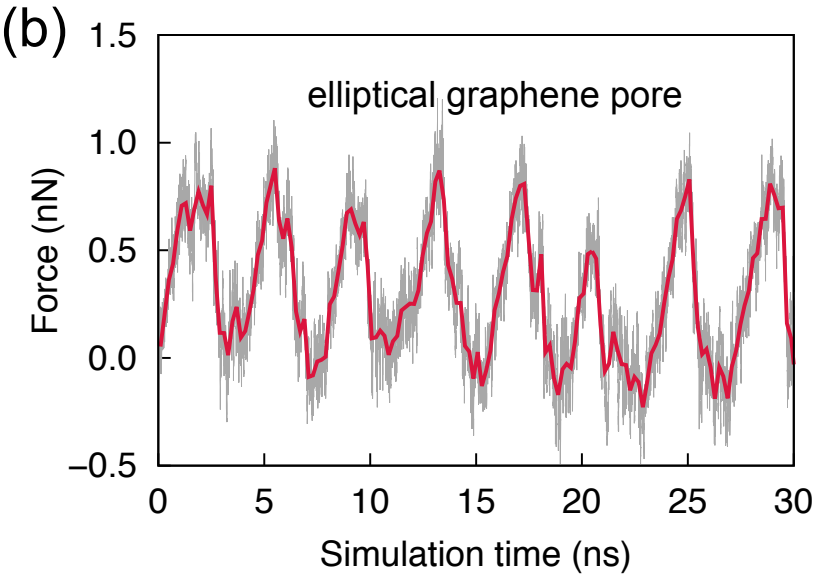

(d)
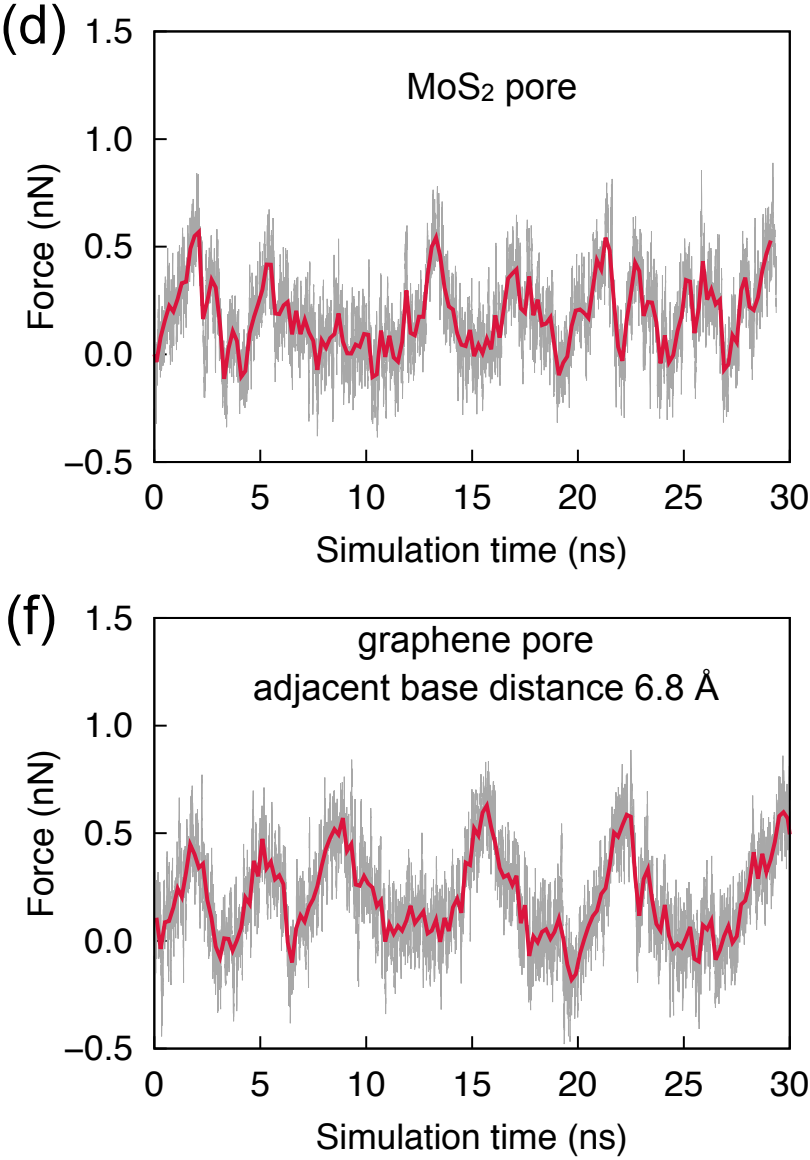

Figure S3: Permeability (left panels) and force signal (right panels) as ssDNA is pulled through an elliptical graphene nanopore $[(\mathrm{a}),(\mathrm{b})]$ and through a circular $\mathrm{MoS}_{2}$ pore of $1.6 \mathrm{~nm}$ diameter $[(\mathrm{c}),(\mathrm{d})]$. The non-bonded interaction of $\mathrm{MoS}_{2}$ were modeled as in a previous study. ${ }^{3}$ (e),(f) Permeability and force signal for a less-stretched ssDNA, namely stretched to a distance between adjacent bases of $0.68 \mathrm{~nm}$. The data reported in the main text are determined for circular graphene nanopores of $1.6 \mathrm{~nm}$ or $2.4 \mathrm{~nm}$ diameter and for ssDNA stretched to a $0.77 \mathrm{~nm}$ distance between adjacent bases. Skipping events in nucleotide permeation, in which multiple bases permeate the nanopore simultaneously, are indicated by arrows. 


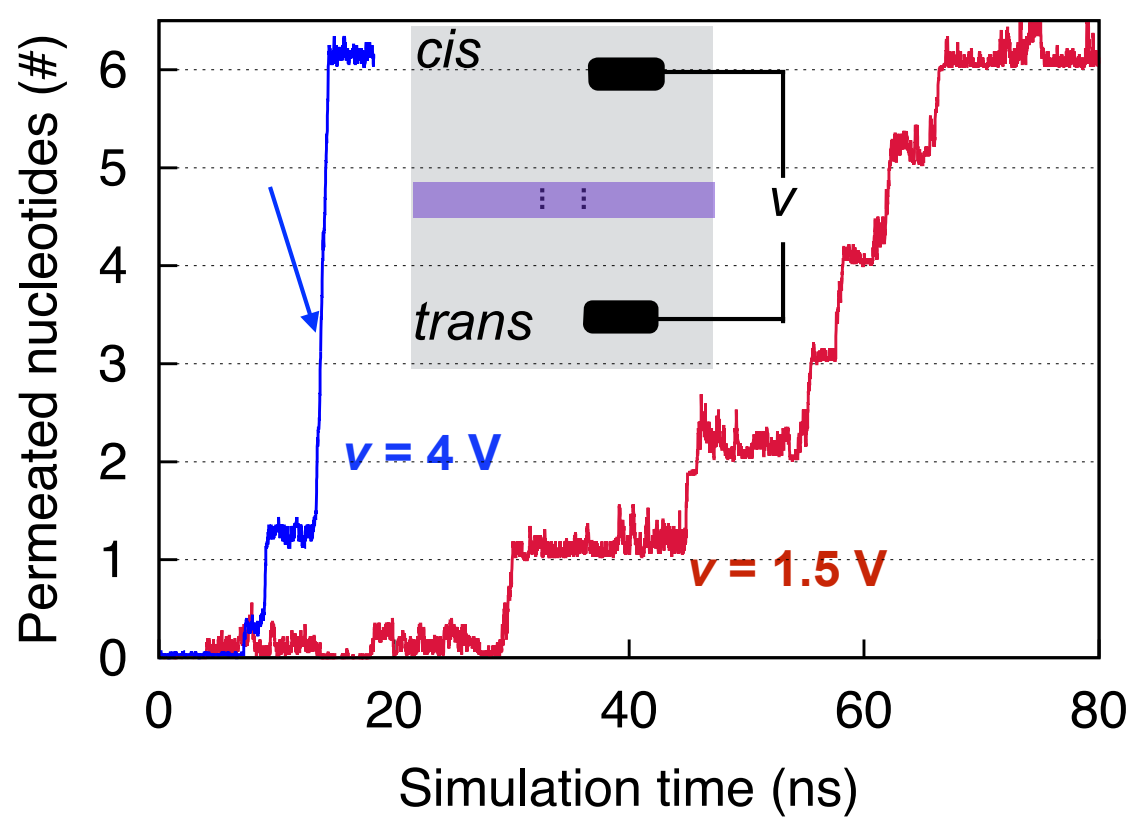

Figure S4: Permeability of stretched ssDNA translocating through a $1.6 \mathrm{~nm}$ diameter graphene nanopore driven by bias voltages of $1.5 \mathrm{~V}$ and $4 \mathrm{~V}$. Inset shows schematic of DNA nanopore translocation. 


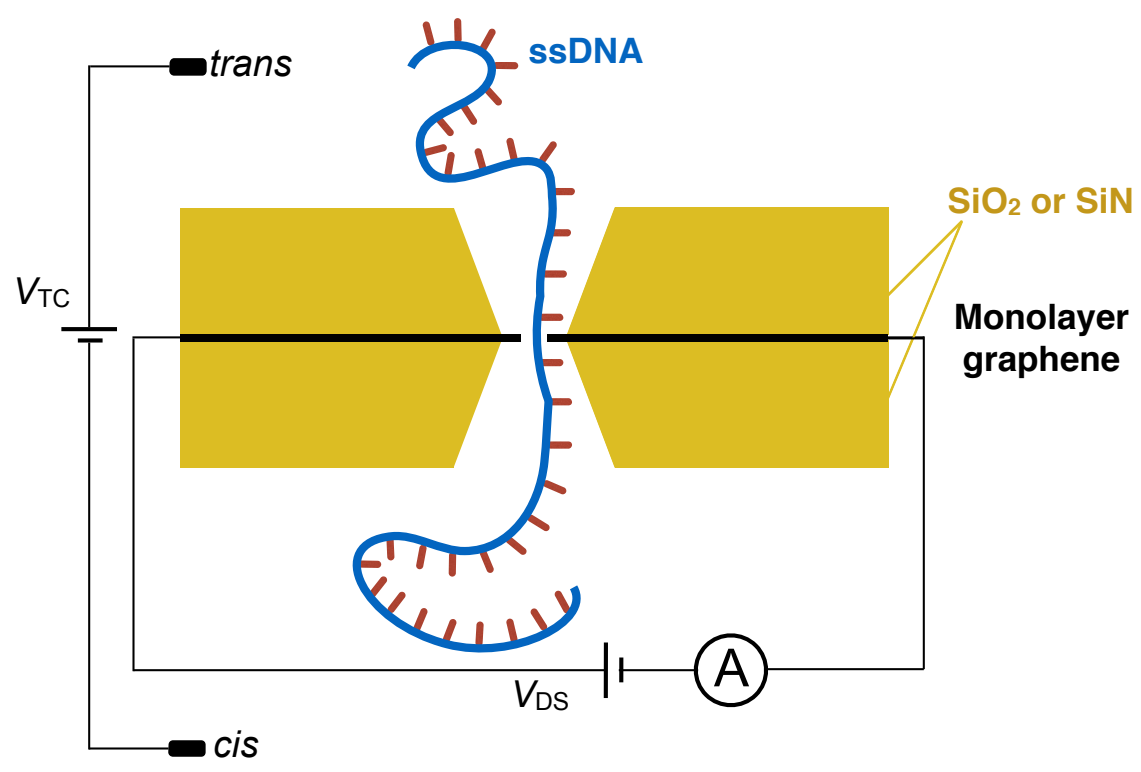

Figure S5: Schematic illustration of a proposed multilayer nanopore device to stretch, translocate and sense ssDNA simultaneously. The device consists of a monolayer graphene sheet sandwiched between two layers of thicker solid-state material such as $\mathrm{SiO}_{2}$ and $\mathrm{SiN}$. The rim of the graphene nanopore is exposed to the pore volume to interact with DNA bases to produce the stepwise DNA translocation reported in this study. The stretching of DNA molecules inside nanopores can be readily accomplished by applying an electric field inside nanopores. ${ }^{1}$ The stepwise translocation of DNA through the pores can be driven by a voltage bias, $V_{T C}$. The sensing of DNA is accomplished by measuring the transverse sheet current across the graphene sheet under a source-drain voltage, $V_{D S}$. 


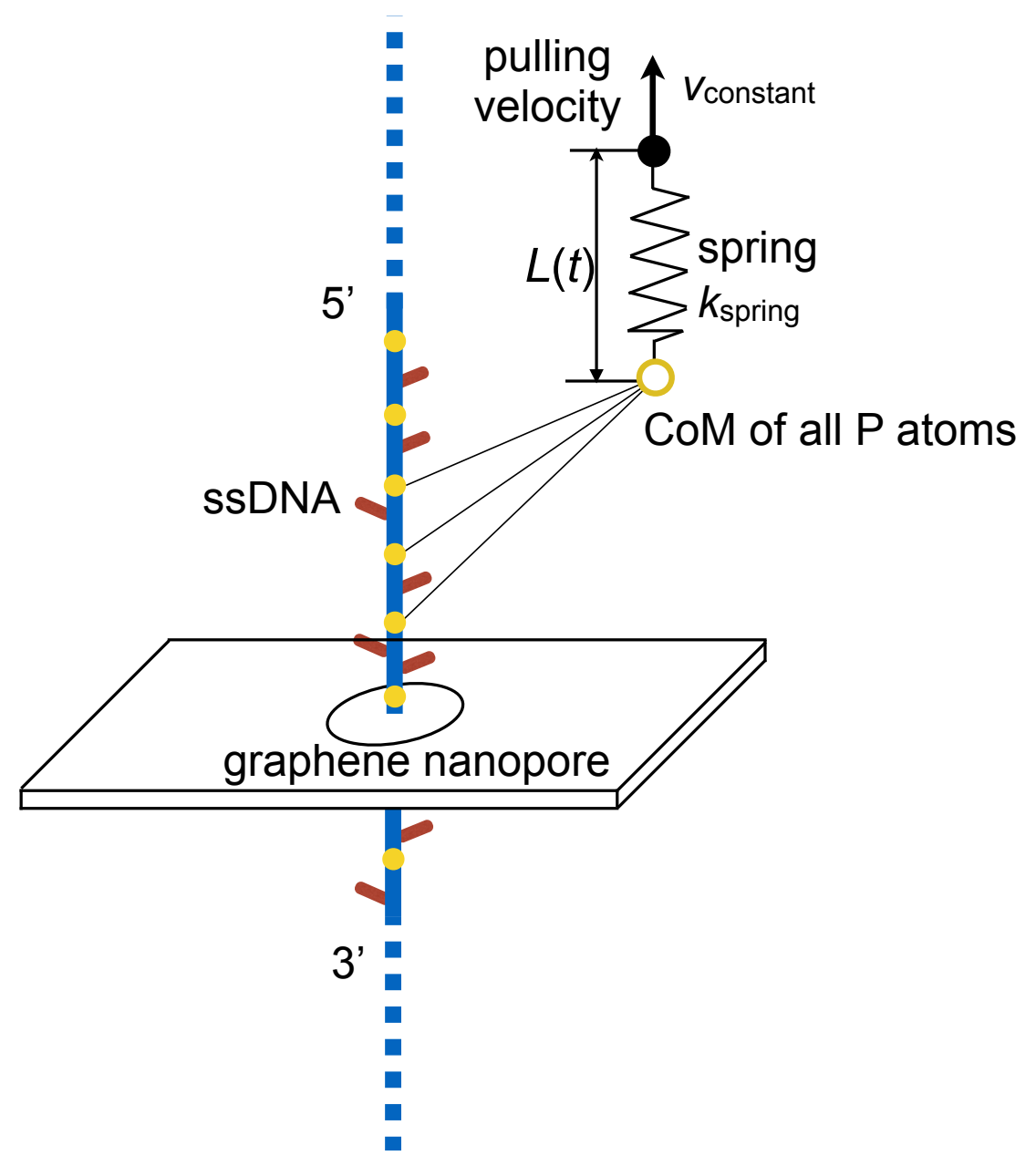

Figure S6: Steered molecular dynamics (SMD) simulations of ssDNA. Aim of the SMD simulations is to pull ssDNA, 5' end first (from bottom to top), through the graphene nanopore. For this purpose a spring is attached with one (first) end to ssDNA and pulled at the other (second) end at constant velocity. The spring elastic property is characterized

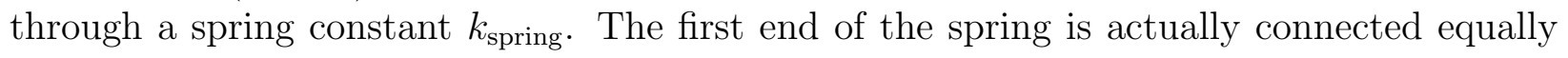
to all phosphorus atoms of ssDNA such that the spring pulls effectively the center of mass (CoM) of the phosphorus atoms. This connection applies the spring force uniformly along the whole ssDNA. As a result of pulling the second end of the spring, ssDNA moves upward through the graphene nanopore. If friction experienced by ssDNA due to ssDNA-graphene interactions would be uniform in time the ssDNA would move with a constant velocity. However, as discussed extensively in the main text, the interactions are not uniform and as a result ssDNA translocation is sometimes slower and sometimes faster. When ssDNA slows down, the length $L(t)$ of the spring extends; when ssDNA moves faster, $L(t)$ decreases. The force acting on the ssDNA is associated with the spring length $L(t)$ through the expression: force $(t)=k_{\text {spring }}\left(L(t)-L_{0}\right)$, where $L_{0}$ is the original spring length. Respective forces are presented in Fig. 3. 


\section{References}

(1) Heng, J. B.; Aksimentiev, A.; Ho, C.; Marks, P.; Grinkova, Y. V.; Sligar, S.; Schulten, K.; Timp, G. Nano Lett. 2005, 5, 1883-1888.

(2) Melnikov, D. V.; Leburton, J.-P.; Gracheva, M. E. Nanotechnology 2012, 23, 255501.

(3) Stewart, J. A.; Spearot, D. Modell. Simul. Mater. Sci. Eng. 2013, 21, 045003. 